\title{
QCD 3 on the world sheet
}

\section{K. Bardakci}

Theoretical Physics Group, Lawrence Berkeley National Laboratory, University of California, Berkeley, California 94720, U.S.A.

E-mail: kbardakci@lbl.gov

ABSTRACT: In this article, we apply the world sheet approach developed in earlier work to QCD in $1+2$ dimensions. The starting point is a field theory on the world sheet that reproduces the planar graphs of QCD parametrized by the light cone variables. We study the ground state of this model using a variational approximation, and show that it consists of a set graphs infinitely dense on the world sheet. The energy of this new ground state is lower than that of the empty world sheet, signaling a phase transition. Also, a finite mass is generated in the originally massless theory. Finally, we consider a particular set of time dependent fluctuations about the static ground state configuration, which result in the formation of a string on the world sheet.

Keywords: Bosonic Strings, Field Theories in Lower Dimensions, Gauge Symmetry

ARXIV EPRINT: 1905.05294 


\section{Contents}

1 Introduction 1

2 The world sheet picture 3

3 The world sheet field theory 4

$\begin{array}{lll}4 & \text { The setup for the variational calculation } & 6\end{array}$

$\begin{array}{lll}5 & \text { The variational equation for } \mathrm{A} & 7\end{array}$

6 Solution of the recursion relation for the variational states 9

$\begin{array}{lll}7 & \text { The ground state } & 11\end{array}$

8 String formation $\quad 12$

9 Discussion 16

\section{Introduction}

The present article is the continuation of a series of previous articles [1,2], based on the program of the world sheet description of planar graphs of field theories. The field theory studied in the previous work was the $\phi^{3}$ theory, chosen for its simplicity. In the present paper, we apply the methods developed in the earlier work to the more interesting and more physical QCD 3 in $1+2$ dimensions. This model has been studied in the literature extensively using various different approaches [3-10]. The world sheet formulation of QCD we are going to use here was developed in [11-14].

The goal of the program is to sum the planar graphs of a field theory on the world sheet parametrized by the light cone variables [15]. It was shown in [16] that this sum is reproduced by a two dimensional field theory that lives on the world sheet. The world sheet field theories for $\phi^{3}$ as well as for QCD 3 are non-local and somewhat complicated. The challenge is then to find a manageable approximation scheme which captures the essence of the model. The scheme we use in this paper is the well-known variational approach for determining the ground state of a Hamiltonian. With the help of a suitably chosen trial state, we establish the following results for QCD 3:

The ground state of the theory consists of a set of light cone graphs infinitely dense on the world sheet. This is clearly non-perturbative, and in fact, it signals a phase transition triggered by the condensation of the graphs. We also show that in this new phase, the originally massless theory develops a finite mass. 
The ground state is a static (solitonic) configuration. Next, we introduce a particular set of time dependent fluctuations around this static background. These fluctuations restore translation invariance on the world sheet violated by the static background, and they also generate a string on the world sheet. We show this by computing the action that describes these modes.

The following is a preview of the sections of this paper. In section 2, the rules for light cone graphs on the world sheet are reviewed, and the world sheet which reproduces these graphs is described in section 3 . In section 4 , the trial state for the variational calculation is introduced. This state is simple enough to allow explicit calculations, and it also avoids the divergences peculiar to the light cone picture.

In section 5, we derive the variational equations for the trial state, and then partially solve them. The resulting energy of the ground state has a linear divergence in the integral over the transverse momentum $q$. This is due to translation invariance in this variable; the ground state energy is proportional to the volume in the $q$ space. We argue that the relevant finite quantity is then the energy per unit volume.

We note that the ground state energy is negative, lower than the energy of the empty world sheet, which is zero. This signals a phase transition in the model. The interesting physics is then in the spectrum of excitations localized in $q$ above the ground state. These excitations are massive, which means that a finite mass is generated in the originally massless theory.

So far, we have only solved the variational equations partially; the ground state energy has still to be minimized with respect to two remaining parameters $a$ and $\rho_{0}$. In section 6 , we determine the dependence of the energy on these parameters, and in section 7 , we show that the minimum is reached in the limit $a \rightarrow 1$ annd $\rho_{0} \rightarrow \infty$. The crucial parameter $m_{0}$ that charaterizes the local excitations is finite as $\rho_{0} \rightarrow \infty$, but diverges as $g^{2} /(a-1)$ as $a \rightarrow 1$. We argue that there is no adjustable coupling constant in this problem; $g$ merely provides the correct dimension for the energy. We may then let $m_{0}$ instead of $g$ provide this dimension and thereby eliminate the singularity in $a-1$.

In closing this section, we compute the density of the light cone graphs on the world sheet, and find that it goes to $\infty$ as $\rho_{0} \rightarrow \infty$. It is than reasonable to expect that these dense set of graphs will lead to string formation, a problem which we investigate in the next section.

String formation is the result of a particular quantum fluctuation around the original static ground state configuration $[1,2]$. It corresponds to shifting the momentum $q$ by a fluctuating field $v(\tau, \sigma)$. This field can be considered as the collective coordinate which restores the translational symmetry in $q$ broken by the ground state configuration. It will also turn out to be the string coordinate.

In the rest of the section, we compute the contribution of this field to the action, after a somewhat lenghty but straightforward algebra, the result turns out to be the standard string action in the light cone picture. There is, however, a problem: The constant in front of the kinetic energy term is singular in the limit $a \rightarrow 1$ and $\rho_{0} \rightarrow \infty$. This singularity can be eliminated by a suitable scaling of both the coordinate $\sigma$ and the field $v$. After this scaling, the original $\sigma$ interval of finite length becomes an interval of infinite length. We 


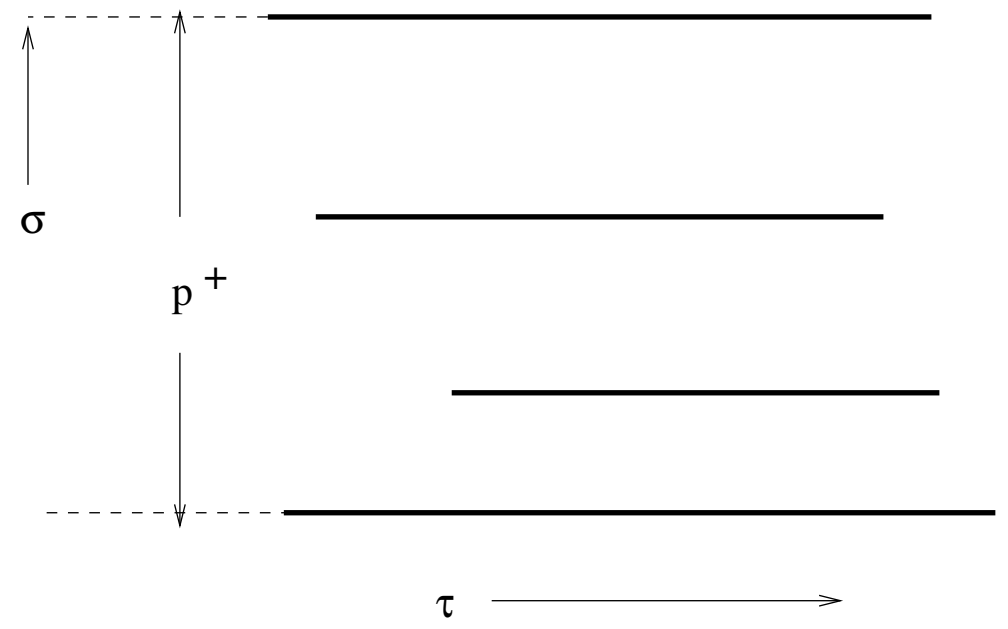

Figure 1. A typical graph.

interpret this as having an action for the bulk of the string, leaving the boundary conditions to be fixed at the end. One can then supply the boundary conditions by cutting a finite piece of the string and glueing the end points together. We should, however, emphasize that the emerging standard string picture is only an approximation, since only the fluctuation of the momentum $q$ was taken into account. The parameters $a$ and $m_{0}$ were fixed and their fluctuations were neglected. Their inclusion would lead to a more complicated string picture, which we hope to investigate in the future.

In the final section, we summarize our results and discuss directions for future research.

\section{The world sheet picture}

The planar graphs of the free part of QCD 3 are the same as in the massless scalar $\phi^{3}$ theory. They can be represented on a world sheet parametrized by the $\tau=x^{+}$and $\sigma=p^{+}$as a collection of horizontal solid lines (figure 1), where the n'th line carries the one dimensional transverse momentum $q_{n}$. Two adjacent solid lines labeled by $\mathrm{n}$ and $\mathrm{n}+1$ correspond to the light cone propagator

$$
\Delta\left(p_{n}\right)=\frac{\theta(\tau)}{2 p^{+}} \exp \left(-i \tau \frac{p_{n}^{2}}{2 p^{+}}\right)
$$

where $p_{n}=q_{n+1}-q_{n}$ is the transverse momentum and

$$
p_{n}^{+}=\sigma_{n+1}-\sigma_{n},
$$

is the light cone momentum flowing through the propagator.

In the interacting theory, in addition to the propagators, there are three and four point vertices. The two three point vertices are pictured in figure 2 . When lines 1 and 2 merge to form the line 3 , the associated vertex factor is given by

$$
V(1+2 \rightarrow 3)=\left(\frac{\sigma_{2}-\sigma_{1}}{\sigma_{3}-\sigma_{2}}+\frac{\sigma_{3}-\sigma_{2}}{\sigma_{3}-\sigma_{1}}\right) p_{2}-\left(\frac{\sigma_{2}-\sigma_{1}}{\sigma_{3}-\sigma_{1}}+\frac{\sigma_{3}-\sigma_{2}}{\sigma_{2}-\sigma_{1}}\right) p_{1}
$$




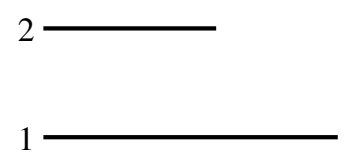

Figure 2. Interaction vertices.

The vertex factor $V(3 \rightarrow 1+2)$, for line 1 splitting into lines 2 and 3 , is given by the conjugate expression. We will not write down the four point vertex since it will not be needed in the present work.

\section{The world sheet field theory}

The light cone graphs described above are generated by a world sheet field theory. We introduce a complex scalar field $\phi(\tau, \sigma, q)$ and its conjugate $\phi^{\dagger}$, which at time $\tau$, annihilate (create) a solid line with coordinate $\sigma$, carrying momentum $q$. They satisfy the usual commutation relations:

$$
\left[\phi(\tau, \sigma, q), \phi^{\dagger}\left(\tau, \sigma^{\prime}, q^{\prime}\right)\right]=\delta\left(\sigma-\sigma^{\prime}\right) \delta\left(q-q^{\prime}\right) .
$$

The vacuum, annihilated by the $\phi$ 's, represents the empty world sheet. For later use, it is also convenient to define the composite operator $\rho$ which represents the density of the solid lines:

$$
\rho^{2}(\tau, \sigma)=\int d q \phi^{\dagger}(\tau, \sigma, q) \phi(\tau, \sigma, q) .
$$

The free Hamiltonian consists of a bunch of solid lines, representing free propagators. An important restriction is that propagators are assigned to adjacent solid lines, and not to the non-adjacent ones. To enforce this constraint, we need to define the projection operator $\mathcal{E}\left(\sigma_{i}, \sigma_{j}\right)$. An explicit construction of this operator in terms of auxilliary fermionic fields was given in [1]. However, this construction is not needed in the present case. All we need to know is the action of this projection operator on the states $|s\rangle$ that will be defined in the next section. This action is defined by the equations

$$
\mathcal{E}\left(\sigma_{i}, \sigma_{j}\right)|s\rangle=0
$$

if $\sigma_{j} \leq \sigma_{i}$.

$$
\mathcal{E}\left(\sigma_{i}, \sigma_{j}\right)|s\rangle=0
$$

if $\sigma_{j}>\sigma_{i}$ and there are solid lines between $\sigma=\sigma_{i}$ and $\sigma=\sigma_{j}$.

$$
\mathcal{E}\left(\sigma_{i}, \sigma_{j}\right)|s\rangle=|s\rangle
$$

if $\sigma_{j}>\sigma_{i}$ and there are no solid lines between $\sigma=\sigma_{i}$ and $\sigma=\sigma_{j}$. These equations are all that is needed to compute the matrix elements $\left\langle s\left|\mathcal{E}\left(\sigma_{i}, \sigma_{j}\right)\right| s\right\rangle$ and derive equations (6.9), (6.15) and (8.8). Also, using the properties of the projection operator described 
above, the free Hamiltonian can be written as

$$
\begin{aligned}
H_{0}= & \frac{1}{2} \int d \sigma \int d \sigma^{\prime} \int d q \int d q^{\prime} \frac{\mathcal{E}\left(\sigma, \sigma^{\prime}\right)}{\sigma^{\prime}-\sigma}\left(q-q^{\prime}\right)^{2} \\
& \times \phi^{\dagger}(\sigma, q) \phi(\sigma, q) \phi^{\dagger}\left(\sigma^{\prime}, q^{\prime}\right) \phi\left(\sigma^{\prime}, q^{\prime}\right) \\
& +\int d \sigma \lambda(\sigma)\left(\int d q \phi^{\dagger}(\sigma, q) \phi(\sigma, q)-\rho^{2}(\sigma)\right),
\end{aligned}
$$

where $\lambda$ is a Lagrange multiplier.

The interaction Hamiltonian, which reproduces vertex factors of (2.2), is given by

$$
\begin{aligned}
H_{I}= & i g \int d \sigma_{1} \int d \sigma_{2} \int d \sigma_{3} \theta\left(\sigma_{2}-\sigma_{1}\right) \theta\left(\sigma_{3}-\sigma_{2}\right) \frac{\mathcal{E}\left(\sigma_{1}, \sigma_{3}\right)}{\sqrt{\left(\sigma_{2}-\sigma_{1}\right)\left(\sigma_{3}-\sigma_{2}\right)\left(\sigma_{3}-\sigma_{1}\right)}} \\
& \times\left(1+\frac{\sigma_{3}-\sigma_{2}}{\sigma_{2}-\sigma_{1}}+\frac{\sigma_{2}-\sigma_{1}}{\sigma_{3}-\sigma_{2}}\right) \rho^{2}\left(\sigma_{1}\right) \rho^{2}\left(\sigma_{3}\right) \int d q_{2} q_{2} \phi\left(\sigma_{2}, q_{2}\right)+\text { H.C. }
\end{aligned}
$$

The $\theta$ functions order the $\sigma$ integrations so that $\sigma_{1}<\sigma_{2}<\sigma_{3}$. The total Hamiltonian

$$
H=H_{0}+H_{I}
$$

as well as the commutation relations (3.1), follow from the action

$$
S=\int d \tau\left(i \int d \sigma \int d q \phi^{\dagger} \partial_{\tau} \phi-H(\tau)\right)
$$

An important feature of this action is its symmetries. It is invariant under the light cone subgroup of Lorentz transformations, and also under translations of the transverse momentum,

$$
\phi(\tau, \sigma, q) \rightarrow \phi(\tau, \sigma, q+r),
$$

by a constant $r$, as well as translations in $\sigma$ and $\tau$ coordinates. Among the lightcone symmetries, the boost along the special direction 1 is of special importance. Under this transformation, parametrized by $u$, the fields transform as

$$
\phi(\tau, \sigma, q) \rightarrow \sqrt{u} \phi(u \tau, u \sigma, q), \lambda(\tau, \sigma) \rightarrow u \lambda(u \tau, u \sigma), p^{+} \rightarrow \frac{1}{u} p^{+} .
$$

To simplify the algebra, we take advantage of this invariance and set,

$$
p^{+}=1
$$

by taking $u=p^{+}$. The correct $p^{+}$dependence can always be restored at the end of a calculation.

Another important symmetry is

$$
\phi(\tau, \sigma, q) \rightarrow-\phi(\tau, \sigma,-q), \phi^{\dagger}(\tau, \sigma, q) \rightarrow-\phi^{\dagger}(\tau, \sigma,-q) .
$$

These symmetries allow us to simplify the search for the ground state. We follow the common practice and assume that the ground state configuration is invariant under these symmeries. 


\section{The setup for the variational calculation}

In the standard variational approach, the approximate ground state energy and the wave function is computed by sandwiching the Hamiltonian between suitably chosen trial states and minimizing the energy with respect to the variational parameters. In our case, an arbitrary state is generated by applying a product of $\phi^{\dagger}$ 's at various values of $\sigma$ 's and $q$ 's but at a fixed value of $\tau$ to the vacuum. In this section, we will introduce the trial state we will use and carry out part of the variational calculation. The simplest state often used in problems of this type is the coherent state

$$
|c\rangle=N^{-1} \exp \left(\int d \sigma \int d q f(\sigma, q) \phi^{\dagger}(\sigma, q)\right)|0\rangle
$$

where $f$ is the variational function and $\mathrm{N}$ is the normalization constant. This corresponds to a random distribution of solid lines with no correlations between them. In our case, there is a problem with using this state: When two adjacent lines with coordinates $\sigma_{1}$ and $\sigma_{2}$ approach each other,

$$
\sigma_{2}-\sigma_{1} \rightarrow 0
$$

the Hamiltonian (3.3), (3.4), becomes singular, and the variational energy blows up. This can be traced back to the $1 / p^{+}$factors in (2.1), typical of the light cone picture. The simplest way to avoid this blowup is to introduce correlation functions between adjacent solid lines which vanish sufficently fast as the lines approach each other. This is a general feature of quantum mechanics: At the locations where the potential becomes singular, the wave function has to vanish. Accordingly, we define the auxiliary states by the recursion relation

$$
|n+1, \sigma\rangle=K(\sigma) \int_{0}^{\sigma} d \sigma^{\prime} g\left(\sigma-\sigma^{\prime}\right)\left|n, \sigma^{\prime}\right\rangle,
$$

and the initial condition

$$
|n=0, \sigma\rangle=|0\rangle,
$$

where $n$ is a positive integer and $\sigma$ ranges from 0 to $p^{+}=1$.

For the correlation function $g$, we choose

$$
g\left(\sigma-\sigma^{\prime}\right)=\left(\sigma-\sigma^{\prime}\right)^{a / 2},
$$

where $a$ is a positive variational parameter. For sufficently large $a$, there is no energy blowup. This choice is motivated by the simplicity of this ansatz, which makes it possible to compute various quantities explicitly.

We take for $K$

$$
K(\sigma)=\int d q A(\sigma, q) \phi^{\dagger}(\sigma, q),
$$

and the variational state is then given by

$$
|s\rangle=\sum_{n=2}^{\infty}|n, \sigma=1\rangle .
$$


We note that in this ansatz, the dependence on $q$ and $\sigma$ factorizes. It is then easy to show that the contribution of the four point vertex vanishes. This is, of course, a feature of this particular ansatz and is not true in general.

This variational state is closely related to the coherent state. In fact, for

$$
a=0
$$

it is the coherent state with

$$
f(\sigma, q)=A(\sigma, q)
$$

We also note that sum over $n$ starts at $n=2$ instead of $n=0$, so that, empty world sheet and a single line are eliminated.

We now have to compute the normalized expectation value of the Hamiltonian,

$$
\langle H\rangle \equiv N^{-1}\langle s|H| s\rangle,
$$

as a function of $A, \rho$ and $\lambda$, the variational parameters of the problem, and solve the corresponding variational equations. We designate the solutions to these equations by a subscript 0 , and take $A_{0}(q), \lambda_{0}$ and $\rho_{0}$ to be independent of $\sigma$. This is because the ground state wavefunction is expected to be invariant under the symmetries of the problem, in this case, translation invariance in $\sigma$. We will discuss invariance under translations of $q$ later on. From now on, we will use the notation

$$
\langle O\rangle=N^{-1}\langle s|O| s\rangle
$$

for the normalized expectation value of any operator $O$.

In the next section, we will solve the variational equation for $A$, and leave the rest to the subsequent sections.

\section{The variational equation for $\mathrm{A}$}

By sandwiching $H$ between the states $|s\rangle$, it is easy to show that the normalized expectation values of the various terms of the Hamiltonian are of the form

$$
\begin{aligned}
& \left\langle H_{0}\right\rangle=Z_{0} \int d q q^{2}|A(q)|^{2}, \\
& \left\langle H_{I}\right\rangle=i g Z_{I} \int d q q\left(A(q)-A^{\star}(q)\right),
\end{aligned}
$$

and,

$$
\langle H\rangle=\left\langle H_{0}\right\rangle+\left\langle H_{I}\right\rangle+\lambda_{0}\left(\int d q|A(q)|^{2}-\rho_{0}^{2}\right) .
$$

$Z_{0}$ and $Z_{I}$ are functions of $\rho_{0}$, to be determined in the next section. Here, we have imposed a constraint on $A(q)$, which we then integrate over. This introduces two new parameters $\lambda_{0}$ and $\rho_{0}$, which prove very useful in simplifying the calculations. Also, taking advantage of translation invariance in $\sigma$, all of the variational parameters are taken to be independent 
of this variable. To save writing, $\rho$ and $\lambda$ are replaced by their ground state expectation values $\rho_{0}$ and $\lambda_{0}$, to be determined later.

The variational equation,

$$
\frac{\delta\langle H\rangle}{\delta A^{\star}(q)}=Z_{0} q^{2} A(q)+\lambda_{0} A(q)-i g Z_{I} q=0,
$$

has the solution

$$
A(q)=A_{0}(q)=i g Z_{I} \frac{q}{Z_{0} q^{2}+\lambda_{0}}
$$

From

$$
\int d q\left|A_{0}(q)\right|^{2}=\rho_{0}^{2}
$$

it follows that

$$
\lambda_{0}=\frac{\pi^{2}}{4} g^{4} Z_{I}^{4} Z_{0}^{-3} \rho_{0}^{-4}
$$

Both $A_{0}(q)$ and $\lambda_{0}$ being fixed, the only variables left are $a$ and $\rho_{0}$. Later, we are going to expand $A$ around $A_{0}(q)$ (5.4). It is important to notice that such an expansion always starts with quadratic terms. All linear terms, in particular the interaction term, are eliminated.

If we now try to compute $\langle H\rangle$ using (5.4), we find that the integral over $q$ is linearly divergent. This is due to the translation invariance in $q$ : in a translationally invariant dynamical problem, energy is proportional to the volume, so the finite quantity is the energy density. The solution for $A_{0}(q)$ may not look translationally invariant, but for large $|q|, q A_{0}(q)$ goes to a constant:

$$
q A_{0}(q) \rightarrow i g Z_{I} / Z_{0} \text {. }
$$

As a result, if we put the system in a one dimensional box of size $L$, the leading term in $\langle H\rangle$ is proportional to $L$ for large $L$, and the energy density $E$ is given by

$$
E=\frac{\langle H\rangle}{L} \rightarrow-g^{2} \frac{Z_{I}^{2}}{Z_{0}}
$$

We note that $E$ is negative. This is the sign of a phase transition: The energy of this new state is lower than the energy of the former ground state, the vacuum, which is zero. Of course only the energy differences matter: We can add a constant and set the energy of the new state equal to zero, and the energy of the vacuum would then be positive.

Ultimately, we are interested in fluctuations local in $q$ around the translationally invariant ground state. These are generated by fluctuations in $A_{0}(q)$ that vanish rapidly for large $|q|$. If we set

$$
A(q)=A_{0}(q)+\nabla A(q),
$$

where the fluctuation term $\nabla A(q)$ vanishes for large $|q|, H$ can be expanded to second order as

$$
H \rightarrow\langle H\rangle+\int d q Z_{0}\left(q^{2}+m_{0}^{2}\right)(\nabla A(q))^{2} .
$$

Here, we have defined a mass parameter by

$$
m_{0}^{2}=\frac{\lambda_{0}}{Z_{0}}
$$


From the structure of the fluctuation term, it is clear that the originally massless theory now has a mass. In fact, this could already been seen by writing $A_{0}(q)$ as

$$
A_{0}(q)=i g \frac{Z_{I}}{Z_{0}} \frac{q}{q^{2}+m_{0}^{2}}
$$

However, so far we have only solved the variational equation for $A(q)$. It is possible that the solution of variational equation for $\rho_{0}$ leads to a vanishing mass. To find the solution to this equation, we first need to compute $N, Z_{0}$ and $Z_{I}$ as functions of $\rho_{0}$. We do this in the next section by solving the recursion relation (4.2).

\section{Solution of the recursion relation for the variational states}

We start with the normalization constant for the variational state $|s\rangle(4.6)$ :

$$
N=\langle s \mid s\rangle=\sum_{n=2}^{\infty}\langle n, \sigma=1 \mid n, \sigma=1\rangle=\sum_{n=2}^{\infty} N(n, \sigma=1)
$$

where,

$$
N(n, \sigma)=\langle n, \sigma \mid n, \sigma\rangle .
$$

The recursion relation (4.2) for the auxiliary states can be rewritten as

$$
|n+1, \sigma\rangle=\int d q \int_{0}^{\sigma} d \sigma^{\prime}\left(\sigma-\sigma^{\prime}\right)^{a / 2} A_{0}(q) \phi^{\dagger}(\sigma, q)\left|n, \sigma^{\prime}\right\rangle
$$

and the corresponding recursion relation for $N(n, \sigma)$ is

$$
N(n+1, \sigma)=\rho_{0}^{2} \int_{0}^{\sigma} d \sigma^{\prime}\left(\sigma-\sigma^{\prime}\right)^{a} N\left(n, \sigma^{\prime}\right) .
$$

By either Fourier or Laplace transforming in the variable $\sigma$, this is reduced to an algebraic equation, which is easily solved. The result can be written as

$$
N(n, \sigma)=\rho_{0}^{2 n} \sigma^{n(1+a)-1} \frac{(\Gamma(1+a))^{n}}{\Gamma(n(a+1)}
$$

and therefore,

$$
N=\sum_{n=2}^{\infty} \rho_{0}^{2 n} \frac{(\Gamma(1+a))^{n}}{\Gamma(n(a+1)}
$$

where eq. (6.1) has been used.

In deriving this result, we have imposed periodic boundary conditions at the end points $\sigma=0$ and $\sigma=1$. This is a convenient choice, although it will become clear later that the precise form of the boundary conditions are not important, so long as translation invariance in the $\sigma$ variable is not violated. In the end, it is only the bulk that matters.

Next, we will consider the matrix elements of $H_{0}$, which can be written as

$$
\left\langle s\left|H_{0}\right| s\right\rangle=M_{0}\left(\rho_{0}\right) \int d q q^{2}\left|A_{0}(q)\right|^{2},
$$


with,

$$
Z_{0}=\frac{M_{0}}{N}
$$

$M_{0}$ is then given by

$$
M_{0}=\rho_{0}^{2} \int_{0}^{1} d \sigma^{\prime} \int_{0}^{\sigma^{\prime}} d \sigma\left\langle s\left|\frac{\mathcal{E}\left(\sigma, \sigma^{\prime}\right)}{\sigma^{\prime}-\sigma}\right| s\right\rangle .
$$

Referring to eq. (6.3), the calculation of the right hand side of this equation is similar to the calculation of $N$, except for the factor $1 /\left(\sigma^{\prime}-\sigma\right)$ multiplying $\mathcal{E}\left(\sigma, \sigma^{\prime}\right)$. This factor can be inserted in the state $|n, 1\rangle$ in $n+1$ different ways, and the resulting $n+1$ terms are all identical because of the periodic boundary conditions. We therefore compute the term say for $\sigma^{\prime}=1$ and multiply the result by $n+1$. Combining these observations, we have

$$
M_{0}=\int_{0}^{1} d \sigma(1-\sigma)^{a-1} \sum_{n=1}^{\infty}(n+1) N(n, \sigma),
$$

and substituting for $N(n, \sigma)$ from (6.4) and doing the integral over $\sigma$ gives

$$
M_{0}=\sum_{n=1}^{\infty}(n+1) \rho_{0}^{2 n} \frac{\Gamma(a)(\Gamma(1+a))^{n}}{\Gamma(a+n(1+a))} .
$$

Next, we evaluate the matrix elements of the interaction Hamiltonian:

$$
\left\langle s\left|H_{I}\right| s\right\rangle=\sum_{n=2}^{\infty}\left\langle n, 1\left|H_{I}\right| n+1,1\right\rangle+\text { H.C. }
$$

From the structure of the vertex (2.2) (figure 2), this calculation involves the integral

$$
\begin{aligned}
\text { Int. } & =\int_{\sigma_{1}}^{\sigma_{3}} d \sigma_{2}\left(\left(\sigma_{2}-\sigma_{1}\right)\left(\sigma_{3}-\sigma_{2}\right)\left(\sigma_{3}-\sigma_{1}\right)\right)^{(a-1) / 2}\left(1+\frac{\sigma_{3}-\sigma_{2}}{\sigma_{2}-\sigma_{1}}+\frac{\sigma_{2}-\sigma_{1}}{\sigma_{3}-\sigma_{2}}\right) \\
& =T\left(\sigma_{3}-\sigma_{1}\right)^{a^{\prime}-1}
\end{aligned}
$$

where $a^{\prime}=(3 a+1) / 2$ and,

$$
T=\frac{3 a+1}{a-1} \frac{(\Gamma((a+1) / 2))^{2}}{\Gamma(a+1)} .
$$

The matrix elemet of $H_{I}$ can now be written as

$$
\left\langle s\left|H_{I}\right| s\right\rangle=i g T M_{I} \rho_{0}^{2} \int d q q\left(A_{0}(q)-A_{0}^{\star}(q)\right),
$$

where,

$$
\begin{aligned}
M_{I} & =\int_{0}^{1} d \sigma \sigma^{a^{\prime}-1} \sum_{n=1}^{\infty}(n+1) N(n, \sigma), \\
& =\sum_{n=1}^{\infty}(n+1) \rho_{0}^{2 n} \frac{\Gamma\left(a^{\prime}\right)(\Gamma(a+1))^{n}}{\Gamma\left(a^{\prime}+n(a+1)\right)} .
\end{aligned}
$$


We note that eq. (6.16) is almost the same as eq. (6.9), except that the exponent of $\sigma$ is now $a^{\prime}-1$, instead of $a-1$. With this replacement, the derivation of (6.16) goes through exactly as in the case of (6.5). Putting together eqs. (5.1) and (6.15), we have,

$$
Z_{I}=N^{-1} \rho_{0}^{2} T M_{I}
$$

We also note that $T$ and therefore $Z_{I}$ has a factor $1 /(a-1)$, so $a$ has to be greater than one to have a finite result. This explains why the correlation function (4.4) was needed. In the next section, we will use these results to fix the values of $a$ and $\rho_{0}$ for the ground state.

\section{The ground state}

Let us first consider the dependence of $E$ on $a$ for fixed $\rho_{0}$. Because of the factor $1 /(a-1)$ in the definition of $T$ (6.14), as $a \rightarrow 1$,

$$
E \rightarrow-\frac{1}{(a-1)^{2}} \rightarrow-\infty
$$

Therefore, the ground state corresponds to the limit $a \rightarrow 1$. We will set $a-1$, except, of course, in the factor $1 /(a-1)$, and search for a minimum in the variable $\rho_{0}$. At $a=1$, the series for various quantities of interest can be summed in closed form:

$$
\begin{aligned}
N & =\rho_{0} \sinh \left(\rho_{0}\right)-\rho_{0}^{2}, \\
Z_{0} & =\frac{\cosh \left(\rho_{0}\right)-1+\left(\rho_{0} / 2\right) \sinh \left(\rho_{0}\right)}{\rho_{0} \sinh \left(\rho_{0}\right)-\rho_{0}^{2}}, \\
Z_{I} & =\frac{2}{a-1} \frac{\rho_{0} \cosh \left(\rho_{0}\right)-2 \rho_{0}+\sinh \left(\rho_{0}\right)}{\sinh \left(\rho_{0}\right)-\rho_{0}} .
\end{aligned}
$$

Now consider the limit $\rho_{0} \rightarrow \infty$,

$$
Z_{0} \rightarrow \frac{1}{2}, Z_{I} \rightarrow \frac{2 \rho_{0}}{a-1}, E \rightarrow-g^{2} \frac{\rho_{0}^{2}}{(a-1)^{2}}
$$

Clearly, $E \rightarrow-\infty$ as $\rho_{0} \rightarrow \infty$. Therefore, the minimum of $E, E \rightarrow-\infty$, is reached as $a \rightarrow 1$ and $\rho_{0} \rightarrow \infty$, and the ground state values of the two variational parameters are fixed at $a=1$ and $\rho_{0} \rightarrow \infty$. However, this specification may appear somewhat problematic, since it involves asymptotic limits tending to infinity. We will now investigate the ground state in more detail, and show that, with $m$ as the order parameter instead of $\rho$, the ground state will be at some finite $m=m_{0}$.

We start by repeating an earlier observation: Only energy differences are important; so we can adjust the ground state energy to be zero, then the energy of the vacuum and the excitations around it will go to infinity. They will then effectively disappear from the spectrum. It is now important to show that the energies of the local excitations around the ground state are finite. In particular, the generated mass (5.11),

$$
m_{0}^{2}=\frac{\pi^{2}}{4}\left(\frac{g Z_{I}}{\rho_{0} Z_{0}}\right)^{4}
$$


is finite in the limit of infinite $\rho_{0}$. From the asymptotic limits (7.3) for $Z_{0}$ and $Z_{I}$,

$$
m_{0}^{2} \rightarrow 64 \pi^{2} \frac{g^{4}}{(a-1)^{4}},
$$

so, in this limit, $m_{0}^{2}$ is independent of $\rho_{0}$. Therefore, instead of $\rho$, if we take

$$
m^{2}=\frac{\lambda}{Z_{0}}
$$

as the order parameter, the limit $\rho_{0} \rightarrow \infty$ can be replaced by $m^{2}=m_{0}^{2}$.

From eq. (7.5), it looks like $m_{0}^{2}$ is singular at $a=1$. However, this is if $g$ is held fixed in this limit. Instead, if we replace $g$ by $m_{0}^{2}$ through

$$
g^{2}=\frac{(a-1)^{2} m_{0}}{8 \pi}
$$

the singularity at $a=1$ disappears. Are we allowed to do this? After all, there is no adjustable coupling constant in this problem; $g$ is there merely to provide the correct dimension for the energy. If we let, instead of $g, m_{0}^{2}$ provide this dimension, the $a \rightarrow 1$ limit can be taken without any blowup.

What does the ground state look like? We will now show that the world sheet is covered by a network of infinitely dense solid lines, corresponding to an infinitely dense set of light cone graphs. We start by noticing that in the series for $N(6.5)$, the summation index $n$ corresponds to the number of solid lines. The n'th term in this series is proportional to the relative probability for the occurence of $n$ lines. Denoting by $\langle n\rangle$ the average value of $n$ weighed by these probabilities, we have,

$$
\langle n\rangle=\frac{1}{2 N} \rho_{0} \frac{\partial N}{\partial \rho_{0}} .
$$

Substituting the large $\rho_{0}$ limit (7.2) for $N$ gives

$$
\langle n\rangle \rightarrow \frac{\rho_{0}}{2} \rightarrow \infty
$$

as $\rho_{0} \rightarrow \infty$. This then shows that the ground state corresponds to an infinitely dense set of graphs on the world sheet. It is then quite plausible that these graphs will form a string of some kind $[17,18]$. In the next section, we will show that this is indeed what happens.

What is the reason for the condensation of the world sheet graphs? It is easy to see from the Hamiltonian that the lines of a graph actually repel each other, so where does the attraction that causes condensation come from? This attraction is provided by entroppy: The entropy of a graph increases exponentially with the number of lines. What we have here is the standard picture for phase transition; entropy balances short range repulsion.

\section{String formation}

To recapitulate, the ground state corresponds to the configuration

$$
i \phi_{i}=A_{0}(q), \phi_{r}=0, a=1, m^{2}=m_{0}^{2},
$$


where $\phi_{r, i}$ are the real and imaginay parts of $\phi$, and $A_{0}(q)$ is given by eq. (5.12). We will now consider time dependent fluctuations around this static configuration. The particular fluctuation that leads to string formation corresponds to shifting the momentum $q$ by the fluctuating field $v(\tau, \sigma)$. We therefore start by letting

$$
i \phi_{i} \rightarrow A_{0}(q+v(\tau, \sigma)) .
$$

In addition, $\phi_{r}$ is taken to be non-zero, and with $q$ again shifted by $v(\tau, \sigma)$ :

$$
\phi_{r} \rightarrow \phi_{r}(\tau, \sigma, q+v(\tau, \sigma))
$$

We will see later that a non-zero $\phi_{r}$ is needed to have the correct canonical quantization of the fields.

$A_{0}(q)$ originally broke translation invariance in $q$, since it was localized around $q=0$. The introduction of the collective coordinate $v(\tau, \sigma)$ restores it, since

$$
q \rightarrow q+r
$$

will be accompanied by

$$
v \rightarrow v-r .
$$

$v$ is then the Goldstone mode of the symmetry generated by translations in $q$. It will also turn out to be the string coordinate. In this paper, we will only consider fluctuations generated by $v$, with $a$ and $m^{2}$ fixed at their ground state values given by (8.1). Fluctuations in these parameters will hopefully be studied in a future work.

If the ansatz given by (8.2) and (8.3) for $\phi_{i}$ and $\phi_{r}$ are substituted in the kinetic energy term in the action (3.6), this term becomes,

$$
\begin{aligned}
K . E . & =-2 \int d \tau \int d \sigma \int d q \phi_{r}(\tau, \sigma, q+v(\tau, \sigma)) \partial_{\tau} \phi_{i}(\tau, \sigma, q+v(\tau, \sigma)) \\
& \rightarrow 2 i \int d \tau \int d \sigma \int d q \phi_{r}(\tau, \sigma, q+v(\tau, \sigma)) \partial_{\tau} A_{0}(q+v(\tau, \sigma)) \\
& =-2 g \frac{Z_{I}}{Z_{0}} \int d \tau \int d \sigma \int d q \phi_{r}(\tau, \sigma, q) \partial_{\tau} v(\tau, \sigma) \partial_{q}\left(\frac{q}{q^{2}+m_{0}^{2}}\right) .
\end{aligned}
$$

Here we have an action first order in the time $(\tau)$ variable, with $\phi_{r}$ and $v$ as conjugate canonical variables. This was the reason for keeping a non-zero $\phi_{r}$. Later, $\phi_{r}$ will be eliminated using its equations of motion, and resulting action will depend only on $v$.

Next we consider the fluctuations of $\langle H\rangle$, which all come from $\left\langle H_{0}\right\rangle$. As explained earlier, the expansion of $A(q)$ around $A_{0}(q)$ eliminates the interaction term. We then make the replacement given by (8.2) and (8.3), and then change the variable of integration from $q$ to $q-v(\tau, \sigma)$. The result is

$$
\begin{aligned}
\langle H\rangle \rightarrow & \frac{1}{2} \int d \sigma \int d \sigma^{\prime} \int d q \int d q^{\prime}\left\langle\frac{\mathcal{E}\left(\sigma, \sigma^{\prime}\right)}{\sigma^{\prime}-\sigma}\right\rangle\left(q-q^{\prime}+v\left(\sigma^{\prime}\right)-v(\sigma)\right)^{2} \\
& \times \phi^{\dagger} \phi(\sigma, q) \phi^{\dagger} \phi\left(\sigma^{\prime}, q^{\prime}\right)+\int d \sigma \lambda(\sigma)\left(\int d q \phi^{\dagger} \phi(\sigma, q)-\rho^{2}\right) .
\end{aligned}
$$


Expanding in powers of $q$ and $q^{\prime}$, terms linear in $q$ and $q^{\prime}$ involve the integral

$$
\int d q q \phi^{\dagger} \phi(\sigma, q)=0
$$

which vanishes because of the symmetry (3.10). We can therefore set,

$$
\langle H\rangle=\langle\tilde{H}\rangle+\left\langle H_{v}\right\rangle
$$

where $\langle\tilde{H}\rangle$ is $v$ independent and $\left\langle H_{v}\right\rangle$ is quadratic in $v$ :

$$
\begin{aligned}
\left\langle H_{v}\right\rangle & =\frac{1}{2} \int d \sigma \int d \sigma^{\prime} \int d q \int d q^{\prime}\left\langle\frac{\mathcal{E}\left(\sigma, \sigma^{\prime}\right)}{\sigma^{\prime}-\sigma}\right\rangle\left(v(\sigma)-v\left(\sigma^{\prime}\right)\right)^{2} \phi^{\dagger} \phi(\sigma, q) \phi^{\dagger} \phi\left(\sigma^{\prime}, q^{\prime}\right) \\
& =\frac{1}{2} \rho_{0}^{4} \int d \sigma \int d \sigma^{\prime}\left\langle\frac{\mathcal{E}\left(\sigma, \sigma^{\prime}\right)}{\sigma^{\prime}-\sigma}\right\rangle\left(v(\sigma)-v\left(\sigma^{\prime}\right)\right)^{2} .
\end{aligned}
$$

We now evaluate the right hand side of this equation at $a=1$ and in the limit $\rho_{0} \rightarrow \infty$. First, setting $a=1$ :

$$
\begin{aligned}
\left\langle s\left|\frac{\mathcal{E}\left(\sigma, \sigma^{\prime}\right)}{\sigma^{\prime}-\sigma}\right| s\right\rangle & =\frac{1}{\rho_{0}^{2}} \sum_{n=1}^{\infty} N\left(n, 1-\sigma^{\prime}+\sigma\right) \\
& =\sum_{n=1}^{\infty} \rho_{0}^{2 n-2} \frac{\left(1-\sigma^{\prime}+\sigma\right)^{2 n-1}}{\Gamma(2 n)} \\
& =\frac{1}{\rho_{0}} \sinh \left(\rho_{0}\left(1-\sigma^{\prime}+\sigma\right)\right),
\end{aligned}
$$

and then dividing by $N$ and taking the limit $\rho_{0} \rightarrow \infty$,

$$
\begin{aligned}
\left\langle\frac{\mathcal{E}\left(\sigma, \sigma^{\prime}\right)}{\sigma^{\prime}-\sigma}\right\rangle & =\frac{\sinh \left(\rho_{0}\left(1-\sigma^{\prime}+\sigma\right)\right)}{\rho_{0}^{2} \sinh \left(\rho_{0}\right)-\rho_{0}^{3}} \\
& \rightarrow \frac{1}{\rho_{0}^{2}} \exp \left(-\rho_{0}\left(\sigma^{\prime}-\sigma\right)\right) .
\end{aligned}
$$

Notice that, in the limit $\rho_{0} \rightarrow \infty$, the exponential function is sharply peaked at $\sigma^{\prime}-\sigma=$ 0 . To leading order, all the contribution to the integral comes from the region $\sigma^{\prime}-\sigma \approx 0$, and we can therefore expand $v\left(\sigma^{\prime}\right)-v(\sigma)$ in powers of $\sigma^{\prime}-\sigma$ and keep only the first term:

$$
v\left(\sigma^{\prime}\right)-v(\sigma) \approx\left(\sigma^{\prime}-\sigma\right) \partial_{\sigma} v(\sigma),
$$

with the result,

$$
\begin{aligned}
\left\langle H_{v}\right\rangle & \rightarrow \frac{\rho_{0}^{2}}{2} \int_{0}^{1} d \sigma^{\prime} \int_{0}^{\sigma^{\prime}} d \sigma\left(\sigma^{\prime}-\sigma\right)^{2} \exp \left(\rho_{0}\left(\sigma^{\prime}-\sigma\right)\right)\left(\partial_{\sigma} v(\sigma)\right)^{2} \\
& \rightarrow \frac{1}{\rho_{0}} \int_{0}^{1} d \sigma\left(\partial_{\sigma} v(\sigma)\right)^{2} .
\end{aligned}
$$

Notice that the dependence of $\left\langle H_{v}\right\rangle$ on $v$ is local in $\sigma$. This is in contrast to the original action, which is non-local in $\sigma$. This localization is due to the limit $\rho_{0} \rightarrow \infty$. In this limit, the density of the lines on the world sheet goes to $\infty$, and the distance between 
two adjacent lines goes to zero (see end of section 7). Therefore, the interaction between the world sheet lines becomes local.

Next, we have to compute $\langle\tilde{H}\rangle$ in the same limit of the parameters. $\langle\tilde{H}\rangle$ is given by (8.5), with $v=0$ :

$$
\begin{aligned}
\langle\tilde{H}\rangle= & \int d \sigma^{\prime} \int d \sigma\left\langle\frac{\mathcal{E}\left(\sigma, \sigma^{\prime}\right)}{\sigma^{\prime}-\sigma}\right\rangle \rho_{0}^{2} \int d q q^{2} \phi^{\dagger} \phi(\sigma, q) \\
& +\int d \sigma \lambda_{0}\left(\int d q \phi^{\dagger} \phi(\sigma, q)-\rho_{0}^{2}(\sigma)\right) .
\end{aligned}
$$

Now, in the integral

$$
\int d q q^{2} \phi^{\dagger} \phi(\sigma, q)=\int d q q^{2}\left(\left|A_{0}(q)\right|^{2}+\phi_{r}^{2}(\sigma, q)\right),
$$

the first term on the right was already included in the ground state energy, so it can be dropped, and so,

$$
\begin{aligned}
\langle\tilde{H}\rangle \rightarrow & \int d \sigma^{\prime} \int d \sigma\left\langle\frac{\mathcal{E}\left(\sigma, \sigma^{\prime}\right)}{\sigma^{\prime}-\sigma}\right\rangle \rho^{2}\left(\sigma^{\prime}\right) \int d q q^{2} \phi_{r}^{2}(\sigma, q) \\
& +\lambda_{0} \int d \sigma \int d q \phi_{r}^{2}(\sigma, q)
\end{aligned}
$$

From eqs. (8.8) and (8.9),

$$
\begin{aligned}
& \int d \sigma^{\prime} \int d \sigma\left\langle\frac{\mathcal{E}\left(\sigma, \sigma^{\prime}\right)}{\sigma^{\prime}-\sigma}\right\rangle \rho^{2}\left(\sigma^{\prime}\right) \int d q q^{2} \phi_{r}^{2}(\sigma, q) \\
\rightarrow & \frac{1}{\rho_{0}} \int d \sigma q^{2} \phi_{r}^{2}(\sigma, q),
\end{aligned}
$$

and therefore,

$$
\langle\tilde{H}\rangle \rightarrow \int d \sigma \int d q\left(\frac{q^{2}}{\rho_{0}}+\lambda_{0}\right) \phi_{r}^{2}(\sigma, q) .
$$

The total action is the sum of (8.4), (8.10) and (8.14). The dependence on $\phi_{r}$ in this action can be eliminated using its equations of motion:

$$
\phi_{r}=-g \frac{Z_{I} \rho_{0}}{Z_{0}\left(q^{2}+\lambda_{0} \rho_{0}\right)} \partial_{\tau} v(\tau, \sigma) \partial_{q}\left(\frac{q}{q^{2}+m_{0}^{2}}\right),
$$

and substituting in the action, and expressing $Z_{I}, Z_{0}, g$ and $\lambda_{0}$ in terms of $\rho_{0}$ and $m_{0}$,

$$
S=\int d \tau \int d \sigma\left(\frac{\rho_{0}^{2}}{m_{0}^{4}}\left(\partial_{\tau} v\right)^{2}-\frac{1}{\rho_{0}}\left(\partial_{\sigma} v\right)^{2}\right)
$$

Apart from the normalization factors in front of the two terms, this is the standard string action in the light cone picture. However, these factors present a problem, since they are singular in the limit $\rho_{0} \rightarrow \infty$. This singularity can be eliminated by a rescaling of the sigma coordinate by

$$
\sigma=\left(\rho_{0}\right)^{-3 / 2} \bar{\sigma}
$$


and by defining

$$
v(\tau, \sigma)=\frac{m_{0}^{2}}{\sqrt{2} \rho_{0}} w(\tau, \bar{\sigma}) .
$$

In terms of these new variables, the action becomes,

$$
S=\int d \tau \int d \bar{\sigma}\left(\frac{1}{2}\left(\partial_{\tau} w\right)^{2}-\frac{m_{0}^{4}}{2}\left(\partial_{\bar{\sigma}} w\right)^{2}\right)
$$

Now consider the limits of integration in the new coordinate $\bar{\sigma}$. The limits in $\sigma$ were from 0 to 1 , but for the present purpose, it is better to start with

$$
-\frac{1}{2} \leq \sigma \leq \frac{1}{2}
$$

Then the limits of $\bar{\sigma}$ are

$$
-\frac{1}{2}\left(\rho_{0}\right)^{3 / 2} \leq \bar{\sigma} \leq \frac{1}{2}\left(\rho_{0}\right)^{3 / 2} \rightarrow-\infty \leq \bar{\sigma} \leq+\infty .
$$

What we have here is an action that describes the bulk of an infinitely long string. The boundary conditions are arbitrary, so long as translation invariance in $\bar{\sigma}$ is preserved. However, we know that the glueballs of QCD are the excitations of a closed string, and the action we have describes the bulk of this string without specifying the boundary conditions. We supply the boundary conditions as follows: We cut a piece of the string of length $p^{+}$, where $p^{+}$is the total light cone momentum, end glue the ends together with periodic boundary conditions. Because of translation invariance in $\bar{\sigma}$, the location of the cut does not matter. In the end, we have a string of length $p^{+}$with periodic boundary conditions.

From the foregoing, it may appear that QCD 3 is described by a standard string model. This, however, is an oversimplification: Even in the context of a variational approximation scheme, we have neclected the fluctuations of the variables $a$ and $\rho$. When these are included say in a perturbative scheme, the result will be a more complicated string model. We hope to investigate this model in a future work.

\section{Discussion}

In this article, we have applied the world sheet approach to field theory developed in the earlier work to QCD 3. The main tool is the two dimensional field theory on the world sheet, which sums the planar light cone graphs. We have investigated the ground state of this model using a variational trial state. Several intersting results follow from this calculation. There is a phase transition to a non-perturbative ground state of lower energy than the empty world sheet. This new ground state is generated by an infinitely dense set of light cone graphs, suggesting an emerging string picture. Also, in this new phase a finite mass is generated in the originally massless model. Finally, we have studied a particular fluctuation in the background of the ground state configuration, and showed a standard string in the light cone framework emerges. However, this was done neglecting all other possible fluctuations. A promising future direction of research is to study other important fluctuations, and see how they modify the simple string picture. 


\section{Acknowledgments}

This work was supported by the Director, Office of Science, Office of High Energy of the U.S. Department of Energy under Contract No. DE-AC02-05CH11231.

Open Access. This article is distributed under the terms of the Creative Commons Attribution License (CC-BY 4.0), which permits any use, distribution and reproduction in any medium, provided the original author(s) and source are credited.

\section{References}

[1] K. Bardakci, Scalar Field Theories On The World Sheet: Cutoff Independent Treatment, JHEP 06 (2013) 066 [arXiv: 1304.1466] [INSPIRE].

[2] K. Bardakci, Scalar Field Theories On The World Sheet: A Non-Trivial Ground State, arXiv: 1408.2556 [INSPIRE].

[3] M.B. Halpern, Field Strength Formulation of Quantum Chromodynamics, Phys. Rev. D 16 (1977) 1798 [INSPIRE].

[4] I. Bars and F. Green, Gauge Invariant Quantum Variables in QCD, Nucl. Phys. B 148 (1979) 445 [Erratum ibid. B 155 (1979) 543] [INSPIRE].

[5] J.P. Greensite, Calculation of the Yang-Mills Vacuum Wave Functional, Nucl. Phys. B 158 (1979) 469 [INSPIRE].

[6] M. Bauer and D.Z. Freedman, Spatial geometry of nonAbelian gauge theory in (2+1)-dimensions, Nucl. Phys. B 450 (1995) 209 [hep-th/9505144] [INSPIRE].

[7] O. Ganor and J. Sonnenschein, The 'dual' variables of Yang-Mills theory and local gauge invariant variables, Int. J. Mod. Phys. A 11 (1996) 5701 [hep-th/9507036] [INSPIRE].

[8] D. Karabali and V.P. Nair, A gauge invariant Hamiltonian analysis for nonAbelian gauge theories in (2+1)-dimensions, Nucl. Phys. B 464 (1996) 135 [hep-th/9510157] [INSPIRE].

[9] D. Karabali and V.P. Nair, On the origin of the mass gap for nonAbelian gauge theories in (2+1)-dimensions, Phys. Lett. B 379 (1996) 141 [hep-th/9602155] [INSPIRE].

[10] D. Karabali, C.-j. Kim and V.P. Nair, Planar Yang-Mills theory: Hamiltonian, regulators and mass gap, Nucl. Phys. B 524 (1998) 661 [hep-th/9705087] [INSPIRE].

[11] K. Bardakci and C.B. Thorn, A World sheet description of large $N_{c}$ quantum field theory, Nucl. Phys. B 626 (2002) 287 [hep-th/0110301] [INSPIRE].

[12] C.B. Thorn, A worldsheet description of planar Yang-Mills theory, Nucl. Phys. B 637 (2002) 272 [Erratum ibid. B 648 (2003) 457] [hep-th/0203167] [INSPIRE].

[13] S. Gudmundsson, C.B. Thorn and T.A. Tran, BT world sheet for supersymmetric gauge theories, Nucl. Phys. B 649 (2003) 3 [hep-th/0209102] [InSPIRE].

[14] C.B. Thorn and T.A. Tran, The fishnet as antiferromagnetic phase of world sheet Ising spins, Nucl. Phys. B 677 (2004) 289 [hep-th/0307203] [INSPIRE].

[15] G. 't Hooft, A Planar Diagram Theory for Strong Interactions, Nucl. Phys. B 72 (1974) 461 [INSPIRE]. 
[16] K. Bardakci, A New World Sheet Field Theory, JHEP 10 (2008) 056 [arXiv:0808. 2959] [INSPIRE].

[17] H.B. Nielsen and P. Olesen, A parton view on dual amplitudes, Phys. Lett. 32B (1970) 203 [INSPIRE].

[18] B. Sakita and M.A. Virasoro, Dynamical model of dual amplitudes, Phys. Rev. Lett. 24 (1970) 1146 [INSPIRE]. 